\title{
The effect of abnormal displacement of the retinal image during eye movements'
}

HANS WALLACH ANO CHARLES LEWIS

SWARTHMORE COLLEGE

The displacement of the images on the retina that results from a turning of the eye does not lead to an apparent motion of what is seen. It has been generally assumed that this is due to a compensating process which takes eye movement into account and serves to discount those image displacements that result from eye movements. It follows from this view that an abnormal image displacement, that is, an image displacement that is larger or smaller than the causing eye movement would warrant, should lead to an experienced displacement of the target. Abnormal image displacement was produced by placing the eye in the converging or diverging bundle of rays from a point source that form behind a strong positive lens; this arrangement yielded a disc-shaped image, the projection of the pupil onto the retina, which displaced abnormally during eye movements. By changing the position of the eye along the axis of the lens in relation to the crossing point of the bundle, the degree to which the displacement was abnormal could be varied. For various displacement rates ranging from $25 \%$ to 120 and $400 \%$ of normal, abnormal displacements produced by incidental eye movements remained unnoticed. Only where eye movements were intentional did some of our $S$ s report shifts of the perceived image. It is suggested that the organism copes with the image displacement resulting from the ever-present incidental eye movements not by compensation but by ignoring them.

It is generally assumed that image displacements on the retina which are caused by eye movements do not bring about an apparent displacement of the corresponding perceived objects because a compensating process prevents this. ${ }^{2}$ The problem is analogous to one raised by head movements. They produce a displacement between the visual environment and the head which does not lead to a perceived displacement of the environment. It is supposed that the effect in the nervous system of those displacements of the environment (relative to the head or the eyes) which are caused by the perceiver's movements is cancelled by messages carrying information about these movements.

That such compensation operates in the case of head movements cannot be in doubt. Wallach \& Kravitz (1965) have recently measured the accuracy with which it operates by displacing a target in various amounts during and dependent on head movements. An apparatus that put the displacement of a target under the control of the S'S head movements made it possible to have a projected light spot displace in any desired ratio to the head's rotation. It consisted of a variable transmission whose input chaft was attached to S's head and whose output shaft controlled the beam that projected the target spot by means of a mirror. When $S$ turned his head back and forth the target moved from left to right and back, and the extent of the target shift as well as its sense (that is, whether the target moved in the direction of the head rotation or against it) was dependent on the setting of the transmission. By reading the transmission setting, the current ratio of target displacement to head rotation could be ascertained. With the help of this apparatus it was found that for most Ss only small displacements of the target during head movements were compatible with it being seen as stationary, i.e., small relative to the angular displacement of the head. Displacements of the target larger than those making up this "no-motion range" were clearly perceived as target motion. By determining the range of displacement ratios that led to perceived target rest, the authors were able to measure this no-motion range. Expressed in percent of the displacement ratio (DR) of 1.0 (where the target was displaced by the same angle as the head) the no-motion range amounted on the average to less than $6.6 \% \mathrm{DR}$.

It was the original purpose of the work now to be reported to make analogous measurements for eye movements. We asked: how much may a target object be displaced during an eye movement and appear to remain stationary? In connection with head movements, target displacement during and dependent on a head rotation can be obtained in two ways: with the help of a variable transmission as just mentioned, or with certain optical devices such as reversing or magnifying goggles. These devices produce optical displacements of objective directions with head rotation and thus simulate motion of the target object during head movements. But when such devices are attached to the head they do not produce target displacements dependent on eye movements. Only if these optical devices were moving with the eyes (as in the other case they are moving with the head) would optical target displacements be abnormal and would displacements of target objects be simulated. An analogue to the variable transmission used to present objective target displacement during head movements would require that $\mathrm{S}$ wear a contact lens and this would have limited severely the number of Ss which could have been employed.

We therefore investigated the effect of target displacement during eye movement without an external target. Instead we used an arrangement which produced directly a retinal image that had the property of being abnormally displaced when the eye turned. In other words, this image shifted during an eye movement not 
A

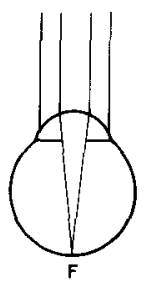

B

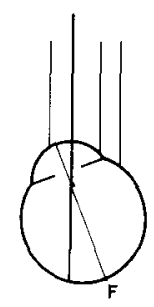

II
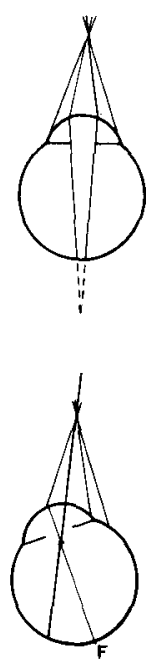

III
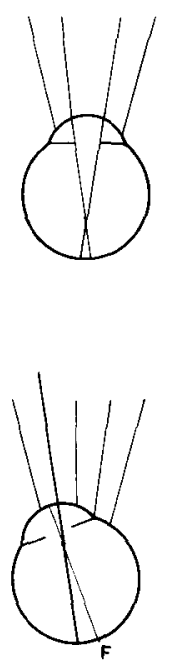

Top: The eye is directed forward. Rays intercepted by the pupil are shown for the eye looking at a distant target (1), or when the eye's optical axis coincides with the axis of the lens (II and III).

Below: The eye is turned to the left. Only the ray through the eye's optical center is shown here entering the pupil.

I. The eye receives parallel rays. A. A target image forms on the fovea $(F)$. $B$. The rays entering the pupil still lie in the forward direction; the target image is normally displaced.

II. The eye is in a divergent beam. A. No target image is formed, but the projection of the pupil is centered on the fovea; the target disk's visual direction is forward. $B$. The rays that enter the pupil do no longer lie in the forward direction. The pupil projection is farther from the fovea than the target image is in I-B. The target disk's visual direction is therefore somewhat to the right of forward.

III. The eye is in a convergent beam. A. Again only the projection of the pupil forms on the retina, here after rays have crossed. $B$. The rays entering the pupil do no longer come from straight ahead, but arrive somewhat from the left. The pupil projection is nearer to the fovea than the target image in $\mathrm{I}-\mathrm{B}$; it has undergone an abnormal displacement. The simulated target direction is correspondingly altered.

in the manner of an image of a stationary object, but as if it were caused by an object that moved dependent on the eye's rotation. The image that behaved in such a fashion consisted in the projection onto the retina of the eye's pupil, produced by strongly divergent or convergent light. In ordinary vision the eye operates in parallel or slightly divergent light beams. The optical target direction, the direction from which the light from the target enters the eye, remains essentially the same in space when the eye turns (see the drawing). This condition produces normal image displacement. But when, because of its passage through a strong lens, the light from a point source comes to the eye as a strongly divergent or convergent bundle, the eye will intercept rays coming from different directions as it turns; the target image on the retina, if one were formed, would be abnormally displaced. There is, of course, no target image; the eye's refractive apparatus cannot focus converging or strongly diverging light to form an image of its source. Nevertheless, an image comes into existence under these circumstances whose displacement depends on the sequence of directions of the intercepted rays and which is therefore abnormally displaced: the projection of the eye's own pupil formed by the shadow of the iris. It is seen as a luminous disk of indefinite size with a slightly irregular edge and a faint, irregular pattern across its surface. Its location on the retina at any given moment represents, of course, a visual direction, the direction in which a real target, were one to correspond to the pupil projection, would be located. It is, thus, possible to speak of a simulated target direction. Since during an eye movement the pupil projection is abnormally displaced, eye movements will cause dis- placements of the simulated target direction in objective space.

The drawing makes it clear how the simulated target direction changes when the eye is turned in a beam of divergent or convergent light. The central ray that actually enters the eye represents the simulated target direction, namely, the outward projection of the bright retinal image framed by the iris shadow. When, in a divergent beam, the eye is turned to the left, the pupil intercepts rays which are progressively more slanted to the right, and this means that the direction of the simulated target is being displaced to the right. Thus, the simulated target displacement is here opposite to the eye movement. When the eye is turned left in a convergent beam, the light entering the pupil arrives more and more from the left; the direction of the simulated target is displaced with the eye movement. When a convex lens is used, which produces not only a convergent beam but, beyond the point where the rays cross, a divergent one also, it is the location of this crossing point in relation to the pupil which matters. It determines whether the sense of the simulated target displacement is with or opposite to the eye movement. The nearer the crossing point is to the plane of the pupil, the larger is the simulated target displacement relative to the eye movement that caused it, that is, the greater is the displacement ratio. The crossing point may fall, in fact, inside the eye.

When the size of the pupil projection is an issue, the eye's own refractive power need be considered. Under ordinary conditions, when the eye intercepts nearly parallel light beams, the eye's focal point falls, of course, on the retina. In convergent or strongly diver- 
gent light the crossing point forms in front of or behind the retina (see drawing), and the projection of the pupil is seen instead of the light source. If the crossing point forms far from the retina, the projection of the pupil may be quite large, particularly when the crossing point is near the plane of the pupil; the projection will then cover a good part of the retina and be useless. But even a moderately large size, which is associated with lenseye distances that yield high displacement ratios (DR), may interfere with our experiment; the targetdisk often changed shape when the eye was moved. We therefore diminished the actual pupil size by administering a contracting drug (pilocarpine, $2 \%$ solution) when the lens setting was for a high $\mathrm{DR}$.

\section{Apparatus}

The position of the eye in relation to the lens was secured by a strong bite board which by means of a sturdy mounting was fixed to a heavy table. The bite board consisted of a $1 / 8$ in metal plate heavily coated with an impression compound which was molded for the individual $S$ to fit his teeth, molars included. The compound lens was fastened from below to the horizontal arm of an adjustable stand, which enabled $\mathrm{E}$ to align the lens' axis with S's right eye and to alter its distance from the eye. In most cases, the lens was an Erfle eye piece of $5 \mathrm{~cm}$ diam. and $3.7 \mathrm{~cm} \mathrm{f.}{ }^{3}$ A point source of light $.5 \mathrm{~mm}$ across was placed on the optical axis of the lens with its distance from the lens adjustable.

A thin glass plate 1 in square was inserted between the lens and S's eye. To his right at a distance of $50 \mathrm{~cm}$ were three signal lights in the shape of thin vertical lines $5 \mathrm{~mm}$ long, each of a different color. These lines were visible to $\mathrm{S}$ by reflection in the glass plate which was appropriately tilted. They were mounted on a pair of horizontal guide rods so that $\mathrm{S}$ could slide them back and forth. Mirrored in the glass they appeared straight ahead, superimposed on the target disk. Since they were objectively given, their images were, of course, normally displaced during eye movements. These lights were used to measure the abnormal displacement of the target disk, and were lit only while measurements were being made.

It was important that during his observations $\mathrm{S}$ saw only the luminous disk; any other light spot that would remain stationary during eye movements would have served as a frame of reference for the change of the disk's visual direction. The darkening of the room was complete and reflections of the light source at the inner surface of the lens mounting were eliminated. The intensity of the light source itself was kept low to prevent light scattered inside the lens from being seen; it would then have formed a faintly visible disk outlined by the edge of the lens.

\section{Procedure}

Because of the novelty of our technique we made no atter. + to set the apparatus accurately for particular displacement ratios. Rather, the particular combination of distances between light source, lens and eye to be offered was based on an "educated guess," to be followed later by the empirical measurements of DR to be described. Inasmuch as preliminary experiments had shown that subjecting $S$ to the measurement procedure sometimes alters the result of subsequent observations, the measuring was done only after Shad made the observations our experiment called for. ${ }^{3}$ The measurements were made in the following manner: First $S$ was instructed to look at the right pole of the target disk and to bring one of the signal lines (A) to coincide with it, by sliding it along the guide rods. Then $S$ was asked to move another signal line (B) and make it coincide with the left pole of the target disk. He was to do this while fixating the right pole which was now marked with the colored line (A). (The distance between these two signal lines defines the visual angle of the target disk.) Now he was asked to look directly at the second signal line. As a result of this eye movement to the left, the target disk was abnormally displaced and therefore changed its position in relation to the signal lines. When the pupil intercepted convergent light from the lens, the target disk was displaced to the left, and when it intercepted divergent light, the disk shifted to the right. While $S$ fixated the second colored line (B) which no longer coincided with the left pole of the disk, he brought a third signal light (C) to coincide with this pole. This concluded S's part in measuring the abnormal displacement. It remained for $\mathbf{E}$ to read the distances between the signal lines on a scale that was attached to the guide rods. The distance between lines $A$ and $B$ represented the angle of the eye movement and the distance between lines $\mathrm{B}$ and $\mathrm{C}$ the degree to which the displacement of the target disk was abnormal, that is, the angle of the simulated displacement that the target disk underwent during the eye movement. The fraction $\mathrm{BC} / \mathrm{AB}$ therefore represented the displacement ratio (DR), the ratio of the angular displacement that the simulated target direction underwent during an eye movement to the angle through which the eye had turned. This procedure of measuring DR was often varied in minor ways, either to make it easier for S (particularly when the target disk was large or the abnormal displacement great), or to measure the effect of the same lens setting with different methods. Such repeated measurements for the same lens setting showed good agreement. It may be mentioned that a displacement ratio of $100 \%$, when the simulated target displacement is with the eye movement, is that of a stabilized image. It remains to be seen whether our arrangement can be adapted to yield accurate stabilization.

Preliminary experiments had shown that the type of measurement Wallach and Kravitz had made in the case of target displacement during head movement could not be done for eye movements. The majority of Ss were oblivious to simulated target displacements of any amount during incidental eye movements and, with intentional eye movements, reports of apparent target motion were so erratic that it seemed impossible to determine 
a no-motion range. We therefore made it our goal to demonstrate the essential lack of sensitivity to abnormal image displacement during eye movements. For this purpose an $\mathrm{S}$ made two observations, one with incidental eye movements, the other while moving his eyes intentionally. By incidental eye movements we mean those saccadic movements which occur when one looks at something and of which one is normally unaware. The usual instructions for observing with incidental eye movements were: "Check the edge of the disk for sharpness on all sides." Only in a few cases were these instructions changed, namely, where we aimed for large displacement ratios (actually where DR was $70 \%$ or greater). Here the disk was quite large and a large eye movement would have shifted it so far to the side that its edge would have become deformed. Ss were therefore asked to inspect the pattern in the disk so that they could describe it. Next, Ss were told: "Move your eyes so that you look from one side of the disk to the other." Those were the instructions meant to elicit intentional eye movements. In those cases where this led to gross deformation of the disk $S$ was asked to make the eye movements smaller but keep looking back and forth. Each observation period lasted from 10 to $15 \mathrm{sec}$, at the end of which $\mathrm{S}$ was asked whether he had seen the disk move.

\section{Results and Discussion}

Having received the instructions meant to elicit incidental eye movements only 4 of our 20 Ss noticed any shifting of the target disk while they inspected it. Of these, two had earlier been exposed to conditions yielding a high DR, had seen the target disk deform and had noticed that eye movements caused the deformations; later, during the critical observation, they claimed to be aware of their eye movements. The third $S$ had also seen the shape distortions but did not report being conscious of eye movements while checking the disk's edge. The fourth of these Ss knew the purpose of the experiment and was aware of eye movements while observing the target disk. The table presents the range of the displacement ratios employed and the distribution of reports of perceived motion. It shows that under the instruction intended to produce incidental eye movements high displacement ratios by no means cause more reports of motion than lower ones. Since 16 out of 20 Ss reported seeing no motion of the target disk and since, of the four who did, three were aware of eye movements during observation, it seems safe to conclude that abnormal image displacement during incidental eye movement normally does not lead to apparent target displacement. Neither will abnormal image displacements regularly do so in the case of intentional eye movements. When Ss were instructed to move their eyes during observation of the target disk, six reported target shifting who had not done so before. But there were still 10 who did not, and four of them had been presented with abnormal image displacement of high DR. Whether or not target shifts are noticed seems to be in part a
TABLE I

Numbers of Ss reporting motion or no motion of the target disk under instruction for incidental or intentional eye movements, grouped for different ranges of displacement ratios (DR).

\begin{tabular}{|c|c|c|c|c|c|c|}
\hline \multirow[t]{2}{*}{$\underline{N}$} & DR range & Av. DR & \multicolumn{2}{|c|}{ Incidental } & \multicolumn{2}{|c|}{ Intentional } \\
\hline & \multicolumn{2}{|c|}{ divergent beam } & no motion & motion & no motion & motion \\
\hline 6 & $28 \cdot 36$ & 35 & 5 & 1 & 4 & 2 \\
\hline \multirow{3}{*}{$\begin{array}{l}4 \\
1\end{array}$} & $50-58$ & 52 & 3 & $1+$ & 3 & 1 \\
\hline & 400 & & 1 & & & 1 \\
\hline & \multicolumn{6}{|c|}{ convergent beam } \\
\hline$\dot{5}$ & $25-47$ & 39 & 3 & $2+$ & 2 & 3 \\
\hline \multirow[t]{2}{*}{4} & $70 \cdot 120$ & 91 & 4 & & 1 & 3 \\
\hline & total & & 16 & 4 & 10 & 10 \\
\hline
\end{tabular}

$+S$ was aware of eye movements

matter of individual differences.

No matter how the 10 cases of reported target motion may be explained, our results are incompatible with the view that the apparent rest of visual objects whose images shift due to eye movements is to be explained by a compensating process which takes the eye movements into account. Even if a very crude process of this sort were operating, at least those of our Ss who were presented with high DR should have reported target shifts when they made incidental eye movements. It rather seems that the nervous system copes with the issue of image displacement due to eye movements by disregarding all image shifts that occur during incidental eye movements, whether they be normal or abnormal. Perceived motion would result from image displacements only when the eye is not actively moving. Neither do we believe that a compensating process is responsible for those cases where abnormal image displacement does lead to an apparent shift of the target disk; instead we think that, where intentional eye movements are concerned, Ss are affected by the altered visual direction of the target disk that results from the eye movement rather than by the abnormal image displacement as such.

This explanation of our results implies that we distinguish between two issues which perhaps are easily considered as one and the same. One is the problem with which our experiment is concerned: Why do objects appear stationary whose images shift on the retina because the eye moves, while image displacement with the eye at restleads to apparent object motion? The second issue concerns the perception of visual direction, which depends on the position of the eye in the head and therefore involves taking the eye position into account. As long as it is assumed that the apparent rest of visual objects during eye movements is due to a process that takes eye movements into account, it is possible to believe that it is the same process that takes eye position into account. But even before our results were obtained it was not a foregone conclusion that the two issues are inseparable. Such a conclusion would imply that perceived motion or rest is solely dependent on change or persistence of visual direction. But this is by 
no means the case; displacement of an object relative to other objects is a much more important factor in motion perception (Duncker 1929, Wallach 1959). Similarly, image displacement in the stationary eye may be a stimulus condition for perceived motion in its own right (rather than by causing a change in visual direction which would, in turn, bring about the motion experience), and a mechanism responsible for the discounting of all image displacements during eye movements may well be concerned with motion perception only. It has never been possible to doubt that the perception of visual direction requires a taking into account of eye position; it is inconceivable that it could occur without such a process, only its manner of operation is a problem. 5 But this is not so with the perceived rest of objects during eye movements. That would be just as effectively achieved if image displacement during eye movements were ignored as if eye movements were taken into account. ${ }^{6}$ The results of our experiment show that image displacement during eye movement is indeed ignored, and this forces us to conclude that the apparent rest of visual objects during eye movements and the perception of visual direction are separate issues.

The separation of the two issues resolves another difficulty, namely, the seeming contradiction between our finding and the result of Wallach and Kravitz. As reported, the latter found rather accurate detection of target displacements that take place during head movements, while our Ss were oblivious to a high degree to target displacement during incidental eye movements. Inasmuch as turning the head always involves incidental eye movements, the results obtained with head movements could not be understood, were we to assume that the present results have a bearing on the perception of visual direction. But if we view our results, as we do, as a matter of motion perception only, the contradiction vanishes.

\section{References}

Duncker, K. Ueber induzierte Bewegung. Psychol. Forsch., 1929, $22,180-259$.

Wallach, H. The perception of motion. Scient. Amer., 1959, 201 No. 1.

Wallach, H., \& Kravitz, J. The measurement of the constancy of visual direction and of its adaptation. Psychon. Sci., 1965, 2, 217-218.

\section{Notes}

1. This work was supported by a grant from the National Science Foundation.

2. For a discussion of this problem, see Teuber, Perception, Handbook of Physiology-Neurophysiology III, p. 1647. A recent exposition is to be found in a chapter by $\mathrm{P}$. A. Merton in The Oculomotor System, ed. Morris Bender, Hoeber Medical Division, Harper and Row 1964, p. 315.

3. When the distance between the eye and the lens had to be short we used our own lens combination of similar properties, which had a larger part of the exit light cone in air.

4. For this reason most ss made observations only for one lens setting; for Ss who made several only one will be reported.

5. Quite recently Matin and Pearce (Science, June 11, 1965, 148, 1485-87) reported an experiment that deals with the dependence of perceived direction on the actual eye position at the time of stimulation. They presented a brief flash at a fixed moment during an eye movement in varying locations and had $\mathbf{S}$ report its apparent position relative to other targets that had previously been visible. Whether or not eye position was taken into account seemed to depend on the time interval between the presentations of the targets whose relative locations were judged.

6. The loss of information due to this "economical" arrangement is negligible. Image displacement as such is a minor factor in ordinary motion perception. which is largely based on the displacement of an image relative to other images (Wallach 1959). Only a brief displacement of the whole visual field or of an object seen against a homogenous environment may remain unnoticed and only if it coincides with the brief time interval occupied by an eye movement. 